\title{
Practice nurse-led proactive care for chronic depression in primary care: a randomised controlled trial
}

\author{
Marta Buszewicz, Mark Griffin, Elaine M. McMahon, Kate Walters and Michael King
}

\section{Background}

Management of long-term depression is a significant problem in primary care populations with considerable on-going morbidity, but few studies have focused on this group.

\section{Aims}

To evaluate whether structured, nurse-led proactive care of patients with chronic depression in primary care improves outcomes.

\section{Method}

Participants with chronic/recurrent major depression or dysthymia were recruited from 42 UK general practices and randomised to general practitioner (GP) treatment as usual or nurse intervention over 2 years (the ProCEED trial, trial registration: ISRCTN36610074).

\section{Results}

In total 282 people received the intervention and there were 276 controls. At 24 months there was no significant improvement in Beck Depression Inventory (BDI-II) score or quality of life (Euroquol-EQ-VAS), but a significant improvement in functional impairment (Work and Social Activity Schedule, WSAS) of 2.5 ( $95 \% \mathrm{Cl} 0.6-4.3, P=0.010)$ in the intervention group. The impact per practice-nurse intervention session was $-0.37(95 \% \mathrm{Cl}-0.68$ to -0.07 , $P=0.017)$ on the BDI-II score and $-0.33(95 \% \mathrm{Cl}-0.55$ to $-0.10, P=0.004)$ on the WSAS score, indicating that attending all 10 intervention sessions could lead to a BDI-II score reduction of 3.7 points compared with controls.

\section{Conclusions}

The intervention improved functioning in these patients, the majority of whom had complex long-term difficulties, but only had a significant impact on depressive symptoms in those engaging with the full intervention.

\section{Declaration of interest}

None.

\section{Copyright and usage}

(c) The Royal college of Psychiatrists 2016.
Depression is an important public health problem, and one of the leading causes of disease burden worldwide. ${ }^{1}$ The Medical Outcomes Study collated data from 11242 out-patients in the USA and showed that depressive symptoms, with or without major depressive disorder, impaired functional ability and wellbeing as much as the most common chronic medical conditions such as diabetes, chronic lung disease, hypertension and heart disease. $^{2,3}$ After 2 years of follow-up around $40 \%$ of those with major depression were still affected and functionally impaired, whereas those with chronic minor depression (dysthymia) had the worst outcome. Most had only partial recovery of functional ability. ${ }^{3}$ Primary care populations with chronic or recurrent depression, although clinically important, are rarely investigated as a distinct patient group. ${ }^{4}$ It is known that chronicity of depression is associated with high mortality, greater psychological and social morbidity and high use of primary care services, ${ }^{5}$ but there is little consistency regarding longer-term management of this disorder. ${ }^{6}$ Studies from the USA indicate that organised, enhanced care can have a beneficial effect on outcomes for patients with acute major depression, ${ }^{7}$ and also those with persistent depression ${ }^{8}$ or at high risk of recurrence. ${ }^{9}$ However, there are increased costs associated with such an approach and beneficial effects can decline over time, such that a longer-term approach may be indicated, particularly for those with chronic difficulties. ${ }^{10}$ Most people with depression are treated in primary care, but there have been few trials of interventions targeted at those with chronic or recurrent depression in either primary or secondary care settings, with most examining interventions for newly diagnosed depression. ${ }^{11}$ Collaborative care models include specialist input, which is potentially more costly to deliver than models based solely in primary care. Given the associated unmet needs, significant morbidity and costs there is a need for new approaches to management of this problem in primary care. The aim of this trial was to evaluate the management of chronic depression with regular structured proactive contact and follow-up of patients by practice nurses over 2 years, supported by general practitioners (GPs) as compared to usual GP care.

\section{Method}

\section{Study design and participants}

This was a randomised controlled trial (RCT) (the ProCEED trial, registered at Current Controlled Trials: ISRCTN36610074) comparing GP 'usual care' (control), with a 'proactive care' approach involving regular follow-up by practice nurses (intervention) in addition to GP usual care, for patients with recurrent or chronic depression (see protocol paper for details ${ }^{12}$ ). Participants were recruited from 42 general practices throughout the UK. All participants gave written, informed consent. Ethics approval was given by the Royal Free Hospital Ethics Committee - REC reference number 07/Q0501/15.

Inclusion criteria were:

(a) adults aged 18 and over;

(b) two or more documented episodes of major depression within the previous 3 years;

(c) evidence of recurrent and/or chronic depression via the Composite International Diagnostic Interview (CIDI, lifetime) questionnaire $^{13}$ (chronic depression was categorised as an episode of major depression lasting at least 2 years within 
the 3 years prior to recruitment or chronic dysthymia for the 2 years prior to recruitment);

(d) a baseline Beck Depression Inventory (BDI-II) score of 14 or above; ${ }^{14}$

(e) sufficient English to be able to complete self-report questionnaires.

Exclusion criteria were:

(a) current psychotic symptoms;

(b) impaired cognitive function;

(c) incapacitating alcohol or drug dependence.

\section{Consent and randomisation}

After a thorough explanation of the study to potential participants, written informed consent was obtained from those fulfilling the inclusion criteria and agreeing to take part. Consenting participants were individually randomised by telephone, using the independent Medical Research Council (MRC) computerised randomisation service and a blocked design to maintain a balance of numbers in control and intervention groups. All participants completed baseline questionnaires at the practice prior to being informed about their randomisation result by the research nurse. All study team members apart from the project manager were masked to block size and group allocation.

\section{Trial intervention}

The intervention, termed 'proactive' care, involved regular scheduled follow-up appointments with trained nurses over the 24 months of the trial. The intervention group had a baseline assessment and further sessions were offered after 1 month, 2 months later and then every 3 months for the remainder of the 2 years of the trial, i.e. a total of 10 appointments. Most were face to face, although patients had the option to elect for telephone appointments when attending the surgery was difficult. A joint management plan was formulated between the nurses and each of their patients at the baseline assessment and reviewed during subsequent appointments. If clinically indicated the reviews could be more frequent and if nurses were concerned about a patient, they were asked to discuss them with the relevant GP, who might also see the patient if indicated.

At each session the nurses asked about the patients' current mood and reviewed their social circumstances, current treatment (medication and/or psychological therapy), and any side-effects. They discussed participants' queries about current or past treatments and checked their concordance with treatment, clarifying any reasons for poor concordance. If there were current symptoms of depression, alternative or additional treatments were discussed. The nurses were given brief training in problem-solving and motivational interviewing techniques ${ }^{15,16}$ and used these to help the participants to identify their own problems, solutions, motivation for change and preferences for care.

The intervention was manualised and has been described in detail elsewhere. ${ }^{12}$ All participating nurses received at least one quality assurance visit from a senior, independent MRC GP research framework training nurse. The intervention group were also given an educational booklet designed for the trial, which included information about depression and that outlined current evidence-based thinking about its treatment (the intervention manual and educational booklet are obtainable on request from the authors).

\section{Nurse training and clinical supervision sessions}

The research team provided 3 days' training for all participating practice nurses. ${ }^{12} \mathrm{~A}$ further day's training was provided for nurses conducting the outcome assessments. Each nurse was assigned a member of the research team as a 'clinical supervisor' (two were GPs with an interest in mental health and one a clinical psychologist). Nurses had telephone contact every 3-4 months with their supervisors and could contact them in between with any patient concerns.

\section{Control arm}

Participants in the control group received 'treatment as usual' and continued to see their GP on request. They did not see the practice research nurse for any mental health intervention.

\section{Outcome measures}

The outcome measures were as follows:

(a) the primary outcome measure was the BDI-II; ${ }^{14}$

(b) functional impairment was measured using the Work and Social Activity Scale (WSAS); ${ }^{17}$

(c) DSM-IV diagnosis and frequency of depressive episodes assessed using the CIDI questionnaire ${ }^{13}$ at recruitment and follow-up;

(d) health-related quality of life measured using the EuroQuol EQ-5D. ${ }^{18}$ Here we report results from the visual analogue scale (EQ-VAS);

(e) practice service data on number of GP visits, practice nurse contacts, referrals for psychological therapy and prescriptions for psychotropic medication collected for 24 months before recruitment and the 24 months of the trial.

The BDI-II was completed at baseline, 3, 6, 12, 18 and 24 months by self-complete questionnaire. All other measures were collected at baseline and 24 months. ${ }^{12}$ The CIDI was completed face to face with a practice research nurse at baseline and 2 years. Final assessment interviews were conducted by research nurses not involved in delivering the intervention who were masked to participants' trial group allocations. As a check on masking they were asked to estimate which trial arm each participant had been randomised into. Practice service-use data were collected by practice nurses involved in the initial recruitment and delivery of the intervention. All other outcome measures were obtained by self-complete questionnaires.

\section{Sample size and statistical analysis}

Data were double entered and analysed using SPSS for Windows release 15.0 and Stata release 10. The sample size was calculated to detect a clinically important difference in BDI-II at $90 \%$ power and the 5\% (two-sided level) of significance. A pooled standard deviation of 11.0 was assumed and the sample sizes adjusted for clustering using an intraclass correlation (ICC) of $0.02 .{ }^{19}$ For a 4-point difference in BDI (assuming an average 10 patients per practice) the required sample size would be $376 .{ }^{12}$ Thus 38 practices with 10 patients per practice (total 380) would meet the required sample size. To take account of possible attrition, recruiting $12-14$ patients per practice would allow for $25 \%$ attrition, meaning a total of 532 participants would be required.

For continuous variables, means and standard deviations were calculated, and for categorical variables, numbers and percentages. All analyses were undertaken on an intention-to-treat (ITT) basis. For the primary (BDI-II) and secondary (WSAS and EQ-VAS) outcomes we used multilevel modelling adjusted for clustering by general practice. ${ }^{20}$ For the BDI-II the multilevel modelling 
included an additional level to take account of repeated measures over time. All models fitted reflected the appropriate hierarchical structure of the data and adjusted for baseline values of the relevant outcome. Practice service-use data were analysed using analysis of covariance (ANCOVA), adjusting for baseline values and using robust standard errors to account for clustering. ${ }^{21}$

To calculate the effect sizes of the main outcome measures we used a method utilising estimates derived from the multilevel models (Hedge's $g$ ). ${ }^{22}$ For the BDI-II, WSAS and EQ-VAS the effect of number of 'nurse sessions' attended on outcome was also assessed using a contamination-adjusted ITT analysis. ${ }^{23}$ This was implemented using instrumental variable regression with the 24month follow-up score as the outcome, randomisation as the instrumental variable, baseline score and number of sessions as explanatory variables and robust standard errors to take account of clustering. A contamination-adjusted ITT analysis allows adjustment for non-adherence, avoiding the biases of as treated and per-protocol analyses and preserving the randomness of allocation by examining how control patients 'would have' behaved were they to have been in the experimental arm. It is similar in principle to a complier average causal effect analysis.

\section{Results}

We approached 3293 potentially eligible people from 42 general practices throughout the UK, identified predominantly from practice-database searches. Participants were recruited between November 2007 and July 2008 and the 2-year follow-up continued until the end of July 2010. Of 3293 people initially approached, 959 (29\%) expressed an interest in attending for interview and $828(25 \%)$ attended. Following the recruitment interview and assessment 558 people were found eligible and agreed to take part (Fig. 1).

Participants' questionnaire responses for the primary outcome, the BDI-II, were: $99 \%$ at baseline; $72 \%$ at 3 months; $66 \%$ at 6 months; $66 \%$ at 12 months; $62 \%$ at 18 months and $78 \%$ at 24 months. Because of concern about attrition between 3 and 6 months, following discussion at the Trial Steering Group and having obtained ethical approval, we incentivised the return of questionnaires from the 12 -month follow-up point with $£ 10$ shopping vouchers. ${ }^{24}$

The 24-month outcome interviews were completed face to face with a different research nurse from the nurse conducting the initial recruitment and intervention and participant response was $65 \%$. A further $13 \%$ of participants returned the final BDI-II by post. A total of 66 participants $(12 \%$ of the total) formally withdrew over the 24 months of the study; 36 in the intervention group and 30 in the control group. One participant in the intervention group died of cancer during the trial.

Of the 282 participants in the intervention group, 77 (27\%) were poor attenders and only attended $0-4$ intervention sessions, $83(29 \%)$ were moderate attenders and attended 5-8 sessions

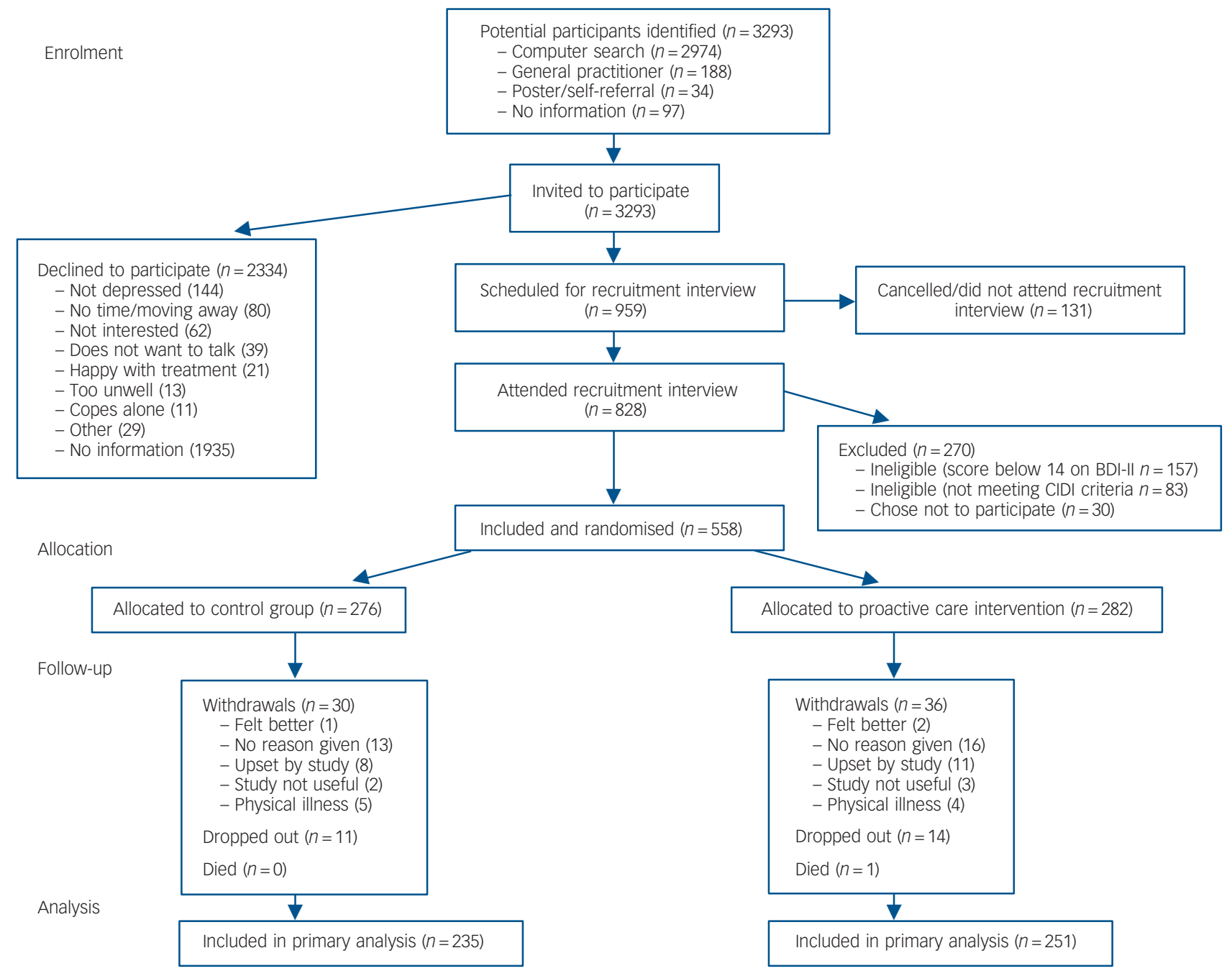

Fig. 1 Consort diagram: recruitment and treatment group allocation. 
and $122(43 \%)$ were good attenders, having attended 9-11 intervention sessions. The latter group were considered to have attended for the full intervention as various timing constraints meant that not all could be offered the full 10 sessions initially intended.

\section{Baseline characteristics}

The two groups were well balanced with respect to baseline characteristics, with no large differences between the intervention and control groups (Table 1).

\section{Outcome results at 24 months}

Primary outcome

Scores for the BDI-II at follow-up improved (decreased) in both groups over time (Table 2). There was a small but not significantly greater improvement in the intervention group. The estimated average difference in score, from the multilevel modelling, was lower (i.e. better) by $1.2(95 \% \mathrm{CI}-0.3$ to $2.7, P=0.125)$ in the intervention group when accounting for all time points.

\section{Secondary outcomes}

Summary results at 24 months are presented in Table 3. From the multilevel modelling, the WSAS score was found to be significantly lower (i.e. better) by 2.5 (95\% CI 0.6 to 4.3 , $P=0.010)$ in the intervention group at 24 months and the
EQ-VAS score was higher (i.e. better) by 2.9 (95\% CI -0.8 to $6.5, P=0.127)$ in the intervention group at 24 months.

The only statistically significant differences in service use were for practice nurse visits and number of months on antidepressants. Both were higher in the intervention group, with adjusted mean differences (intervention-control) of 1.6 (95\% CI 0.2 to $3.0, P=0.026)$ and $1.4(95 \%$ CI 0.02 to $2.8, P=0.047)$ respectively. A comparison of the proportions in each diagnostic category (CIDI) between intervention and control groups at follow-up showed no statistically significant difference $(P=0.368)$.

\section{Effect sizes and contamination-adjusted ITT analysis}

Using Hedge's $g$ method gave us the effect sizes: BDI-II $=0.09$; WSAS $=0.21$; EQVAS $=0.14$. Results investigating the effect of the number of intervention sessions on BDI-II, WSAS and EQ-VAS scores using the contamination-adjusted ITT analysis showed the following 'per nurse treatment-session' effects on average score: BDI-II $(-0.37,95 \%$ CI -0.68 to -0.07 , $P=0.017)$, WSAS $(-0.33,95 \%$ CI -0.55 to $-0.10, P=0.004)$ and EQ-VAS $(0.38,95 \% \mathrm{CI}-0.13$ to $0.88, P=0.142)$, indicating statistically significant improvements (decreases) per session in BDI-II and WSAS scores of -0.37 and -0.33 respectively. We then multiplied the per session effect by the number of sessions attended, which would lead to a reduction in BDI-II score of 3.7 points and a reduction in WSAS scores of 3.3 points more in the patients in the intervention group than controls if all 10 intervention sessions were attended. This assumes from the

Table 1 Baseline sociodemographic characteristics, diagnoses, symptoms, function and health services utilisation ${ }^{\text {a }}$

\begin{tabular}{|c|c|c|c|c|}
\hline & $\begin{array}{l}\text { Intervention } \\
\text { group }\end{array}$ & $\begin{array}{l}\text { Control } \\
\text { group }\end{array}$ & $\begin{array}{l}\text { Intervention } \\
\text { group, } n\end{array}$ & $\begin{array}{l}\text { Control } \\
\text { group, } n\end{array}$ \\
\hline Age, years: mean (s.d.) & $48.3(12.3)$ & $48.4(13.4)$ & 282 & 276 \\
\hline Women, $n(\%)$ & $217(77.0)$ & $201(72.8)$ & 282 & 276 \\
\hline Married, $n(\%)$ & $133(47.7)$ & $127(46.9)$ & 279 & 271 \\
\hline Living with partner/children, $n$ (\%) & $212(76.3)$ & $188(69.1)$ & 278 & 272 \\
\hline Accommodation, owner-occupied, $n(\%)$ & $188(68.6)$ & $179(66.1)$ & 274 & 271 \\
\hline Ethnicity, white: $n$ (\%) & $251(90.6)$ & $241(89.3)$ & 277 & 270 \\
\hline Paid employment, $n$ (\%) & $137(48.9)$ & $121(44.8)$ & 280 & 270 \\
\hline $\begin{array}{l}\text { Diagnosis (Composite International Diagnostic Interview), } n \text { (\%) } \\
\text { Chronic major depression } \\
\text { Recurrent depression } \\
\text { Dysthymia }\end{array}$ & $\begin{array}{r}78(28.1) \\
155(55.8) \\
45(16.2) \\
\end{array}$ & $\begin{array}{r}86(31.6) \\
142(52.2) \\
44(16.2)\end{array}$ & 278 & 272 \\
\hline Beck Depression Inventory-II, mean (s.d.) & $31.9(9.8)$ & $33.1(10.6)$ & 278 & 272 \\
\hline Work and Social Activity Scale, mean (s.d.) & $22.1(9.6)$ & $22.4(9.4)$ & 280 & 272 \\
\hline Euroquol Visual Analogue Scale, mean (s.d.) & $54.5(19.5)$ & $52.8(20.1)$ & 281 & 269 \\
\hline General practitioner visits, mean (s.d.) & $15.5(9.9)$ & $15.8(9.7)$ & 270 & 271 \\
\hline General practitioner home visits, mean (s.d.) & $0.2(1.2)$ & $0.2(0.9)$ & 236 & 224 \\
\hline Practice nurse visits, mean (s.d.) & $3.9(4.7)$ & $4.5(5.1)$ & 258 & 254 \\
\hline Practice counsellor visits, mean (s.d.) & $0.7(2.6)$ & $0.4(1.4)$ & 258 & 254 \\
\hline Referrals to psychological therapy/psychotherapy, mean (s.d.) & $0.4(0.9)$ & $0.3(0.6)$ & 155 & 145 \\
\hline Referrals to psychiatrist/community mental health team, mean (s.d.) & $0.4(0.7)$ & $0.6(1.8)$ & 154 & 154 \\
\hline Number of months on antidepressants, mean (s.d.) & $14.1(8.8)$ & $12.7(8.3)$ & 267 & 269 \\
\hline
\end{tabular}

\begin{tabular}{|c|c|c|c|c|c|c|c|c|c|c|}
\hline & \multicolumn{2}{|c|}{3 months } & \multicolumn{2}{|c|}{6 months } & \multicolumn{2}{|c|}{12 months } & \multicolumn{2}{|c|}{18 months } & \multicolumn{2}{|c|}{24 months } \\
\hline & $n$ & Mean (s.d.) & $n$ & Mean (s.d.) & $n$ & Mean (s.d.) & $n$ & Mean (s.d.) & $n$ & Mean (s.d.) \\
\hline Control group & 180 & $29.2(12.8)$ & 167 & $28.8(13.8)$ & 166 & $27.9(13.6)$ & 152 & $27.3(13.6)$ & 206 & $26.0(14.9)$ \\
\hline Intervention group & 221 & 28.1 (12.3) & 201 & $25.8(12.7)$ & 201 & $25.2(12.8)$ & 196 & 25.1 (14.4) & 224 & $22.1(14.2)$ \\
\hline
\end{tabular}




\begin{tabular}{|c|c|c|c|c|}
\hline & $\begin{array}{l}\text { Intervention } \\
\text { group }\end{array}$ & $\begin{array}{l}\text { Control } \\
\text { group }\end{array}$ & $\begin{array}{l}\text { Intervention } \\
\text { group, } n\end{array}$ & $\begin{array}{l}\text { Control } \\
\text { group, } n\end{array}$ \\
\hline Work and Social Activity Scale, mean (s.d.) & $16.2(12.1)$ & $18.8(12.1)$ & 224 & 205 \\
\hline Euroquol Visual Analogue Scale, mean (s.d.) & $61.7(21.6)$ & $58.0(21.4)$ & 214 & 201 \\
\hline General practitioner visits, mean (s.d.) & $13.7(9.5)$ & $13.4(9.1)$ & 234 & 226 \\
\hline General practitioner home visits, mean (s.d.) & $0.1(0.6)$ & $0.1(0.5)$ & 193 & 190 \\
\hline Practice nurse visits, mean (s.d.) & $5.5(6.6)$ & $4.8(6.6)$ & 234 & 226 \\
\hline Practice counsellor visits, mean (s.d.) & $0.7(2.1)$ & $0.4(1.6)$ & 234 & 226 \\
\hline Referrals to psychological therapy/psychotherapy, mean (s.d.) & $0.6(1.2)$ & $0.3(0.6)$ & 133 & 119 \\
\hline Referrals to psychiatrist/community mental health team, mean (s.d.) & $0.6(1.4)$ & $0.4(0.8)$ & 117 & 126 \\
\hline Number of months on antidepressants, mean (s.d.) & $13.6(9.7)$ & $11.7(9.6)$ & 261 & 250 \\
\hline $\begin{array}{l}\text { Diagnosis (Composite International Diagnostic Interview), } n \text { (\%) } \\
\text { Chronic major depression } \\
\text { Recurrent depression } \\
\text { Dysthymia } \\
\text { No episodes of depression }\end{array}$ & $\begin{array}{l}27(13.8) \\
87(44.4) \\
25(12.8) \\
57(29.1)\end{array}$ & $\begin{array}{l}28(16.6) \\
60(35.5) \\
27(16.0) \\
54(32.0)\end{array}$ & 196 & 169 \\
\hline
\end{tabular}

analysis that each session is likely to have the same effect on outcome and thus the effect of the sessions attended is additive.

\section{Masking of the final assessment}

Agreement between the trial arm allocation and guesses of the research nurses undertaking the final patient assessment $(n=361)$ was low (kappa $(\kappa)=0.281, P<0.001)$, indicating successful masking of the outcome assessments.

\section{Discussion}

\section{Principal findings}

There was no significant improvement in depression score (BDI-II) or quality of life (EQ-5D) at 24-month follow-up in the overall sample, but there was an improvement in social functioning (WSAS). The contamination-adjusted ITT analysis, conducted to assess the effect of the number of sessions received, demonstrated a positive per session effect for both BDI-II and WSAS scores. From this contamination-adjusted ITT analysis it could be inferred that patients attending all 10 intervention sessions might be expected to reduce their BDI-II score by 3.7 points more than control patients. Given the chronicity of this patient group and the severity of their baseline depression and functional impairment, this improvement is encouraging. The higher level of nurse visits in the intervention group was expected, given the nature of the intervention. Both groups had very high levels of GP contact at baseline, which dropped slightly at follow-up, but were not significantly different between the two groups. Antidepressant usage dropped slightly in both groups over the follow-up period, but was significantly higher in the intervention group. There was no evidence that outcome varied by baseline diagnostic group.

\section{Strengths and limitations}

A particular strength was our large, nationally representative sample. The intervention was manualised, straightforward to implement and underwent successful piloting before the trial. Patients were rigorously assessed using standard diagnostic instruments, quality assurance for delivery of the intervention and outcome assessments was ensured and research masking maintained. The study was conducted across 42 UK general practices, but with fewer practices from deprived ethnically diverse inner city areas resulting in a low proportion of participants from
Black and minority ethnic groups, so the results may be less applicable to these populations. Approximately $25 \%$ of patients initially approached attended and completed baseline interviews, which might be a limitation as regards generalisability, but analysis of our baseline data indicated that those participating were a severely affected and highly morbid group. ${ }^{25}$ This was also reflected in their high rates of GP visits at baseline, which were nearly three times higher than general population figures. ${ }^{26}$ There was some attrition over the trial period (65\% completed 24-month assessments), although this is reasonable for this population and similar to other studies. ${ }^{10}$

In our pilot trial there were no identified issues of contamination. There was very little contact between the practice nurses and control patients, and where this occurred they were reviewed exclusively for physical health problems. There may have been a small risk of contamination in the main trial, which if present would lead to an underestimation of the effectiveness of the intervention.

\section{Implications of these findings with reference to other studies}

Our trial focused on the practice nurse as case manager within a chronic care model, with primary care support but, unlike US models of collaborative care, no input from specialist mental health services apart from for individual patients as part of their routine clinical care. This model is similar to that shown to be effective and in widespread use for other long-term conditions such as diabetes and chronic obstructive pulmonary disease, ${ }^{27}$ and could be more easily implemented in a primary care setting using existing staff, such as practice nurses with no previous specific training in mental health. Practice nurses are available in many healthcare systems and achieve good results in managing other long-term conditions. ${ }^{28}$ However, their training has been inadequate for working with people with mental health problems, ${ }^{29}$ which was something we aimed to address with a focused, brief training course and clinical supervision over the study period.

A systematic review of RCTs of case management for depression in primary care highlighted a range of factors likely to be associated with a positive outcome, several of which were present in this trial. ${ }^{30}$ Systematic tracking of patients by a provider other than the doctor was significantly associated with improved depression outcomes and could be further improved by 
incorporating patient preferences into care, which were both factors we included. Practice nurses in our trial received brief training in simple problem-solving and motivational interviewing techniques. ${ }^{15,16}$ Our qualitative evaluation indicated they used a problem-solving approach but made little use of motivational interviewing techniques in delivering the intervention. ${ }^{31}$ They were encouraged not to consider themselves as therapists delivering a psychological intervention, but to refer patients to local psychological therapy services if indicated. However, they reported that access to psychological therapies was often not readily available for their patients, and their role in facilitating access to such treatments was therefore limited. Increased availability of appropriate, evidence-based psychological therapies might have improved patient outcomes.

Our results suggest improvements in functioning were greater than changes in symptoms of depression. This positive impact on functional impairment may be particularly important as there is evidence it is more significant in those with moderate to severe depression. ${ }^{32}$ Whereas functional impairment and disability associated with depression is often noted, there is relatively little emphasis on treatments that may positively have an impact on this. ${ }^{33,34}$ If participants engaged with the intervention and attended all the review sessions they had statistically significant improvements per session in both depressive symptoms and functional impairment. The two are likely to be linked and, although traditionally it is often considered that an improvement in mood leads to improved functioning, the converse may also be the case and it may be that in the context of chronic depression it may be possible to improve functioning without great improvements in depressive symptoms.

The absolute difference in functioning (WSAS) score in our study was modest, and its clinical significance is unclear. However, the results from our linked qualitative study gives support to some patients reporting meaningful changes in functioning resulting from the intervention. ${ }^{35}$ Most participants reported an impact on some aspect of their lifestyle, with the potential impacts on diet, increasing exercise and sleep.

\section{Implications for clinicians and policymakers}

Systematic reviews of RCTs involving patients with depression in primary care or community settings have predominantly included acute cases. ${ }^{11}$ Our population was more chronic and morbid and potentially more difficult to treat, ${ }^{25}$ with very high baseline rates of GP consultations. Practice nurses are a widespread resource and in regular contact with such patients for their physical care, but often feel poorly skilled in working with mental health problems. ${ }^{29}$ The training we provided was very well received ${ }^{31}$ and we would encourage more widespread development of specific, and if possible more intensive, training courses for practice nurses in common mental health problems. We allowed the nurses to decide which approach to take with their patients with reference to the training they had received, but the training was of necessity brief and it would be interesting to see whether a more prescriptive approach to the management of these patients would give different results.

A difficult initial interaction with the nurse, patients who appeared to lack the motivation or time to attend appointments, were reluctant to discuss their difficulties or felt pessimistic that their situation might improve were all factors linked with poor attendance and such patients had poorer outcomes. ${ }^{31}$ It may be appropriate for patients who do not engage well with the practice nurse as case manager to see someone with a mental health background in this role. Our trial suggests participants who engaged well and attended all sessions had more positive outcomes, irrespective of the severity or chronicity of their depression at baseline. We obtained useful information from our qualitative work, suggesting that early development of rapport with the practice nurse and motivation to change were important features of engagement. ${ }^{31}$ This has implications for clinicians considering patient suitability for this type of service.

Our findings indicate that practice nurse-led enhanced care for chronic and recurrent depression shows promise for motivated patients from this highly morbid group in primary care. This model of care may have value in other healthcare systems with limited access to secondary care psychological or psychiatric services and should be evaluated in these settings.

In conclusion, patients with chronic or long-term depression are a neglected group as regards both clinical management and research into effective interventions. In our trial, although overall improvements in depressive symptoms were small and nonsignificant for patients receiving the intervention, there were significant improvements in work and social functioning. Further supplementary analysis indicated patients who regularly attended sessions over 2 years did well, with improvements in both depressive symptoms and functioning. In any implementation, it is key to identify patients more likely to engage with and benefit from such an intervention. The nurses' focus and approach on practical goals and problem-solving may have contributed to the improved levels of functioning obtained.

Marta Buszewicz, MBBS, MRCGP, MRCPsych, Mark Griffin, MSc, Elaine M. McMahon, BA, MPhil, Kate Walters, MBBS, MSC, PhD, Research Department of Primary Care \& Population Health, University College London (Royal Free Campus), London; Michael King, MBBS, PhD, FRCPsych, Division of Psychiatry, Faculty of Brain Sciences, University College London, London, UK

Correspondence: Marta Buszewicz, MBBS, MRCGP, MRCPsych, Research Department of Primary Care \& Population Health, University College London (Royal Free Campus), Upper 3rd Level Rowland Hill Street, London NW3 2PF, UK. Email: m.buszewicz@ucl.ac.uk

First received 24 Jun 2014, final revision 6 Mar 2015, accepted 15 Apr 2015

\section{Funding}

This work was funded by The Big Lottery - grant code RG/1/010166750. We also received the service support costs for which the study was eligible from the Department of Health. The study sponsor was University College London. Neither the sponsor nor the funder had any role in the study design, data collection, data analysis and interpretation, writing of the report or decision to submit for publication.

\section{Acknowledgements}

The authors would like to acknowledge and thank the Big Lottery as the funders of this research and the voluntary organisation Mind who were our collaborators. We would also like to thank the Medical Research Council General Practice Research Framework (MRC GPRF) nurses, participating practices and patients for their involvement, as well as the nurses, practices and patients from the four non-GPRF practices involved. We also received valuable support from the Mental Health Research Network (MHRN) and the Primary Care Research Network (PCRN). We are grateful to Professor Irwin Nazareth and Dr John Cape for their comments on an earlier draft of this paper.

\section{References}

1 Murray CJL, Lopez AD. The Global Burden of Disease: A Comprehensive Assessment of Mortality and Disability from Diseases, Injuries and Risk Factors in 1990 and Projected to 2020. World Health Organization, 1996.

2 Wells KB, Stewart A, Hays RD, Burnam A, Rogers W, Daniels M, et al. The functioning and well-being of depressed patients. Results from the Medical Outcomes Study. JAMA 1989; 262: 914-9.

3 Wells KB, Burnam MA, Rogers W, Hays R, Camp P. The course of depression in adult outpatients. Results from the Medical Outcomes Study. Arch Gen Psychiatry 1992; 49: 788-94.

4 Conradi, $\mathrm{HJ}$, de Jonge $\mathrm{P}$, Ormel J. Prediction of the three-year course of recurrent depression in primary care patients: different risk factors for different outcomes. J Affect Disord 2008; 105: 267-71. 
5 Lloyd KR, Jenkins R, Mann A. Long term outcome of patients with neurotic illness in general practice. BMJ 1996; 313: $26-8$.

6 Arroll B, Moir F. Time for a rethink of treatment for patients with depression in primary care. Br J Gen Pract 2010; 60: 641-2.

7 Katon W, Robinson P, Von Korff M, Lin E, Bush T, Ludman E, et al. A multifaceted intervention to improve treatment of depression in primary care. Arch Gen Psychiatry 1996; 53: 924-32.

8 Simon GE, Katon WJ, VonKorff M, Unützer J, Lin EH, Walker EA, et al. Cost-effectiveness of a collaborative care program for primary care patients with persistent depression. Am J Psychiatry 2001; 158: 1638-44.

9 Katon WJ, Rutter C, Ludman E, von Korff M, Lin E, Simon G, et al. A randomised trial of relapse prevention of depression in primary care. Arch Gen Psychiatry 2001; 58: 241-7.

10 Rost K, Nutting P, Smith JL, Elliott CE, Dickinson M. Managing depression as chronic disease: a randomised trial of ongoing treatment in primary care. BMJ 2003; 325: 934.

11 Gilbody S, Bower P, Fletcher J, Richards D, Sutton A. Collaborative care for depression: a cumulative meta-analysis and review of longer-term outcomes. Arch Intern Med 2006; 166: 2314-21.

12 Buszewicz M, Griffin M, McMahon E, Beecham J, King M. Randomised controlled trial of structured, proactive nurse-led care for chronic depression in primary care: the ProCEED trial. BMC Psychiatry 2010; 10: 61.

13 Kessler RC, Abelson J, Demler O, Escobar Jl, Gibbon M, Guyer ME, et al. Clinical calibration of DSM-IV diagnoses in the World Mental Health (WMH) version of the World Health Organization (WHO) Composite International Diagnostic Interview (WMH-CIDI). Int J Methods Psychiatr Res 2004; 13: 122-39

14 Arnau R, Meagher MW, Norris MP, Bramson R. Psychometric evaluation of the Beck Depression Inventory-II with primary care medical patients. Health Psychol 2001; 20: 112-9.

15 Mynors-Wallis LM, Gath DH, Day A, Baker F. Randomised controlled trial of problem solving treatment, antidepressant medication, and combined treatment for depression in primary care. BMJ 2000; 320: 26-30.

16 Rollnick S, Heather N, Bell A. Negotiating behaviour change in medical settings: the development of brief motivational interviewing. $J$ Ment Health 1992; 1: 25-37.

17 Mundt JC, Marks IM, Shear MK, Greist JM. The Work and Social Adjustment Scale: a simple measure of impairment in functioning. Br J Psychiatry 2002 180: $461-4$

18 Brooks R. EuroQol: the current state of play. Health Policy 1995; 37: 53-72.

19 King M, Davidson O, Taylor F, Haines A, Sharp D, Turner R. Effectiveness of teaching general practitioners skills in brief cognitive behaviour therapy to treat patients with depression: randomised controlled trial. BMJ 2002; 324 947.

20 Rabe-Hesketh S, Skrondal A. Multilevel and Longitudinal Modelling using Stata (2nd edn). Stata Press, 2008.

21 Vickers AJ, Altman DG. Analysing controlled trials with baseline and follow up measurements. BMJ 2001; 323: 1123-4.

22 Hedges LV. Distribution theory for Glass's estimator of effect size and related estimators. J Educ Behav Statist 1981; 6: 107-28.

23 Sussman J, Hayward R. Using instrumental variables to adjust for treatment contamination in randomised controlled trials. BMJ 2010; 340: 1181-84.

24 Edwards PJ, Roberts IG, Clarke MJ, DiGuiseppi C, Wentz R, Kwan I, et al Methods to increase response rates to postal questionnaires. Cochrane Library 2009; 3: MR000008.

25 McMahon EM, Buszewicz M, Griffin M, Beecham J, Bonin E, Rost F, et al. Chronic and recurrent depression in primary care: socio-demographic features, morbidity and costs. Int J Fam Med 2012; 2012: 316-409.

26 Health and Social Care Information Centre. Trends in Consultation Rates in General Practice - 1995-2009. Health and Social Care Information Centre, 2009 (http://www.hscic.gov.uk/pubs/gpcons95-09).

27 Wagner EH, Austin BT, Von Korff M. Organising care for patients with chronic illness. Millbank Q 1996; 74: 511-44.

28 Bodenheimer T, MacGregor $\mathrm{K}$, Stothart $\mathrm{N}$. Nurses as leaders in chronic care. BMJ 2005; 330: 612-3.

29 Crosland A, Kai J. They think they can talk to nurses: practice nurses' views of their roles in caring for mental health problems. Br J Gen Pract 1998; 48: 1383-6.

30 Christensen H, Griffiths K, Gulliver A, Clack D, Kljakovic, Wells L. Models in the delivery of depression care: a systematic review of randomised and controlled intervention trials. BMC Fam Pract 2008; 9: 25

31 Bennett M, Walters K, Drennan V, Buszewicz M. Structured proactive care for chronic depression by practice nurses in primary care: a qualitative evaluation. PLOS One 2013; 8: e75810.

32 Mintz J, Mintz LI, Arruda MJ, Hwang SS. Treatments of depression and the functional capacity to work. Arch Gen Psychiatry 1992; 49: 761-8.

33 Greer TL, Kurian BT, Trivedi MH. Defining and measuring functional recovery from depression. CNS Drugs 2010; 24: 267-84.

34 McKnight PE, Kashdan TB. The importance of functional impairment to mental health outcomes: a case for reassessing our goals in depression treatment research. Clin Psychol Rev 2009: 29: 243.

35 Buszewicz M, Griffin M, Beecham J, Bonin E, Hutson M. ProCEED. Report of a Study of Pro-Active Care by Practice Nurses for People with Anxiety and Depression. Mind Publications, 2011. 